\title{
Spatial anisotropy and heterogeneity in contractility and adhesion distribution may contribute to cell steering during migration
}

\author{
Soumya S S, ${ }^{1}$ Subodh Kolwankar, ${ }^{1}$ Edna George, ${ }^{2}$ Santanu K. Basu, ${ }^{3}$ Shamik Sen, ${ }^{2}$, a) and Mandar M. \\ Inamdar $1, \mathrm{~b})$ \\ 1) Department of Civil Engineering, Indian Institute of Technology Bombay, Mumbai 400076, \\ India \\ ${ }^{2)}$ WRCBB, Department of Biosciences and Bioengineering, Indian Institute of Technology Bombay, \\ Mumbai 400076 India \\ ${ }^{3)}$ Department of Chemical Engineering, Indian Institute of Technology Bombay, \\ Mumbai 400076 India
}

(Dated: 16 July 2018)

Transition from random to persistent cell motility requires spatiotemporal organization of the cytoskeleton and focal adhesions. The influence of these two structures on cell steering can also be gleaned from trypsin de-adhesion experiments, wherein cells exposed to trypsin round up, exhibiting a combination of rotation and translation. Here, we present a model to evaluate the contributions of contractility and bond distribution to experimentally observed de-adhesion. We show that while asymmetry in bond distribution causes only cell translation, a combination of asymmetric bond distribution and non-uniform contractility are required for translation and rotation, and may guide cell migration.

Cell motility is central to various processes including embryogenesis and wound healing. For motility to occur, cells must possess a polarized shape defined by leading and trailing edges, with the cell protruding at the leading edge and retracting at the trailing edge $\underline{1}$. While cell protrusions are stabilized by the formation of integrin-based adhesions, cell retraction requires the detachment of rear adhesions orchestrated by actomyosin-based contractile forces. Efficient migration, therefore, requires close temporal and spatial co-ordination between the cytoskeleton and the adhesion apparatus. On two-dimensional (2-D) substrates, most adherent cells exhibit random cell motility, frequently altering their leading and trailing edges. In contrast, in the presence of directional cues, persistent cell migration is observed $\stackrel{2}{2}$ Since both the cytoskeleton and the adhesions contribute to motility, their relative contributions to such frequent directional changes remain unclear. Moreover, because most mesenchymal cells possess speeds in the order of few microns/hour, for motility to occur, cell shape stability must be maintained at shorter timescales (few minutes) via the force balance between contractile actomyosin forces and the focal adhesions that withstand these forces. This tensional homeostasis can be gauged using the trypsin de-adhesion assay wherein, cell retraction is tracked upon rapidly severing cell-matrix contacts using the enzyme trypsin (Fig. 1 and corresponding Supp. Movie) $\underline{3}$. Further, sigmoidal retraction kinetics with two time constants observed for a wide variety of cells, including fibroblasts, epithelial cells and cancer cells, is indicative of the generality of the process (Fig. 1b).

When adherent $3 \mathrm{~T} 3$ fibroblasts were incubated with the enzyme trypsin, cells exhibited a combination of

\footnotetext{
a) Electronic mail: shamiks@iitb.ac.in

b) Electronic mail: minamdar@civil.iitb.ac.in
}

translation and rotation during the de-adhesion (or rounding up) process (Figs. 1f and 1 d ). Such motions are probably indicative of a combination of anisotropy in cytoskeletal contractile forces (also referred to as cell prestress) and in cell-matrix adhesions (Fig. 1). If such anisotropy exist, this may help us to understand the frequent directional changes associated with random cell motility. In this letter, we specifically address this question by studying the crosstalk between distributions of cell prestress and adhesion on the pattern of de-adhesion exhibited by $3 \mathrm{~T} 3$ fibroblasts. Here, we propose a simple theoretical model which can replicate the above mentioned cell dynamics during the processes of de-adhesion.

To understand the role of various factors dictating deadhesion patterns (Fig. 1 1 and supplementary information (SI) Fig. S1), we began with a $2-\mathrm{D}$ continuum description of a prestressed cell in mechanical equilibrium with its substrate. The cell was modeled as a circle of radius $R_{\text {cell }}$ connected to the substrate by continuous bonds both cell and the substrate were represented as continuous, homogeneous, isotropic visco-elastic Kelvin-Voigt materials 4,5 . The cell-substrate bonds were modeled using linear springs 6 whose number and distribution depended on the type of distribution of focal adhesion between cell and substrate. Since the thickness of the cell is typically small as compared to its other dimensions, it was reasonable to assume condition of plane stress for the present problem $\underline{7}-\underline{\underline{9}}$.

Figures 2 and 2 schematically describe the adhesion and de-adhesion dynamics of the cell. For the purpose of a systematic study, the entire sequence of cell activity was divided into two stages; the initial equilibration stage and the subsequent de-adhesion stage. During the first stage or equilibration stage, the cell which had been initially in a relaxed round shape was brought to a stretched state by the application of specified displacement fields $u_{0}(x, y)$ and $v_{0}(x, y)$ (equivalent to "thermal" prestress ${ }^{7}$ ) 

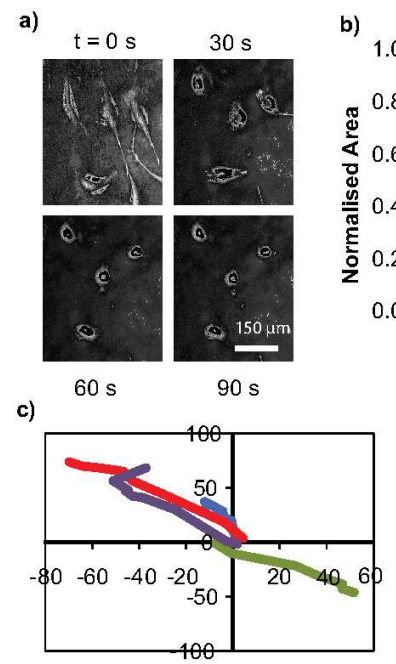

d)

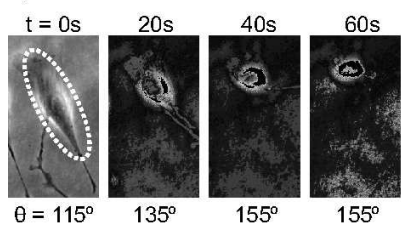

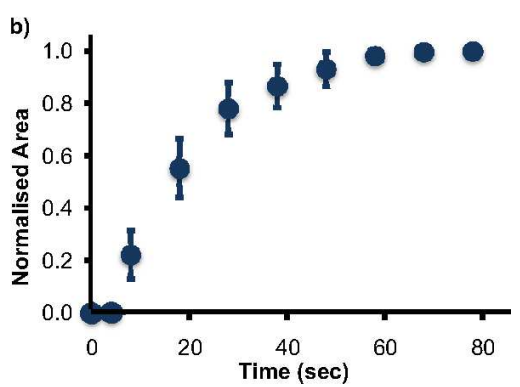

e)

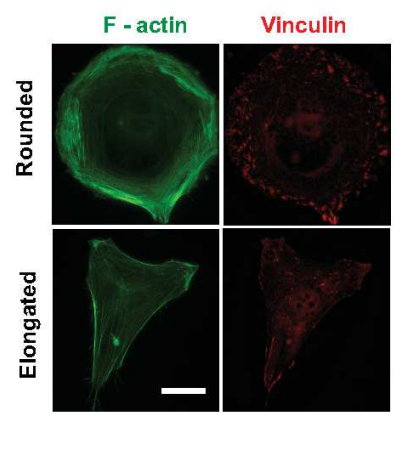

FIG. 1. (Color online) Trypsin induced de-adhesion of NIH 3 T3 fibroblasts. Fibroblasts were cultured on collagen-coated substrates for 24 hours. Before experiment, cells were washed with PBS, warm trypsin was added and images were acquired in time-lapse till cells rounded up but remained attached to the substrate. Also see the corresponding supplementary movie. (a) Sequence of time-lapse images of fibroblasts rounding up upon addition of trypsin. (b) Plot of normalized area (defined as change in area at any time divided by net change in area during the entire de-adhesion process) as a function of time. (c) During de-adhesion, cells underwent both translation and rotation. Quantification of translation obtained by tracking the centroid of individual cells during the time course of de-adhesion. (d) Sequence of images of a cell undergoing rotation during de-adhesion. At each time point, the cell is approximated by an ellipse, with indicating the orientation of the major axis of the cell with the x-axis. (e) Cytoskeletal and focal adhesion organization in cells with round and elongated morphology. Cells were fixed and stained for F-actin (green) for visualizing the actin cytoskeleton and with vinculin (red) for observing the distribution of focal adhesions (enhanced online).

and then attached to the substrate with the help of cellsubstrate bonds. Once the cell was connected to the substrate, it started applying tractions on the substrate and ultimately reached a mechanical equilibrium state with the substrate as shown in Fig. 2a. At this stage, the equation of equilibrium of the system, in terms of displacement components of the cell, $u_{c}$ and $v_{c}$, was

$$
\begin{aligned}
\frac{1}{2(1+\nu)} \nabla^{2} u_{c}+ & \frac{1}{2(1-\nu)} \frac{\partial}{\partial x}\left(\frac{\partial u_{c}}{\partial x}+\frac{\partial v_{c}}{\partial y}\right) \\
& +r_{1} \rho_{0}(x, y)\left(u_{0}-u_{c}\right)=0
\end{aligned}
$$

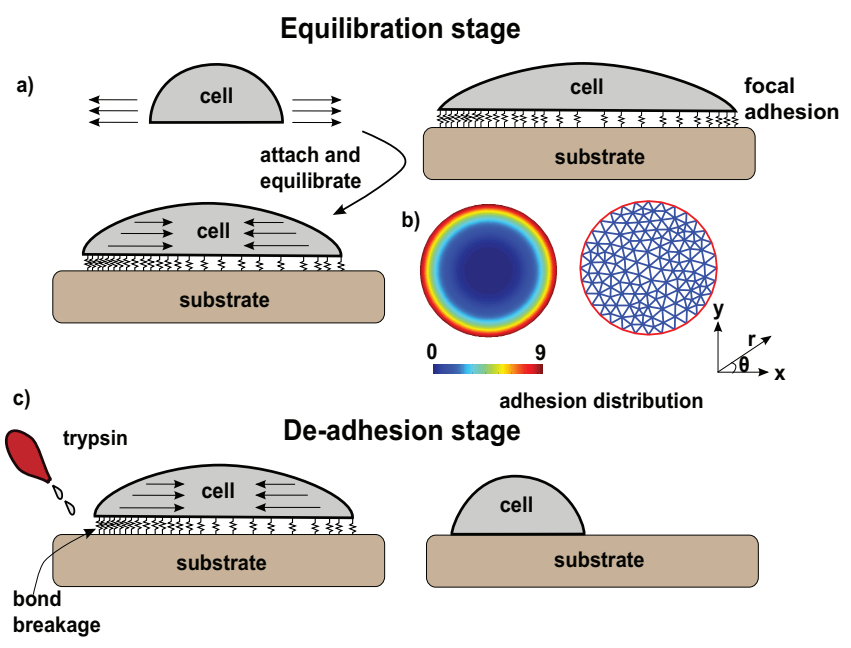

FIG. 2. (Color online) A schematic representation of our modeling of cell adhesion and de-adhesion dynamics. (a) Circular cell was subjected to stretching to mimic contractility. Next, the stretched cell adhered to substrate to represent formation of focal adhesions. Cell then reached a mechanical equilibrium state or tensional homeostasis with substrate by balancing the contractile forces generated by actin-myosin cytoskeleton (inward arrows) and cell-substrate adhesions (springs). (b) To simulate the de-adhesion dynamics of the cell, a finite element analysis was performed. For that, entire domain was discretized into triangular elements. The non uniform distribution of focal adhesion was modeled by varying the number of connected springs over the cell area. (c) De-adhesion process was initiated by adding trypsin to the cell in equilibrium. Upon treatment with trypsin, the focal adhesions were broken in a time dependent manner and cell started to relax. When de-adhesion process was completed, all the focal adhesions were broken and cell attained its original circular shape from the stretched configuration.

$$
\begin{aligned}
\frac{1}{2(1+\nu)} \nabla^{2} v_{c}+ & \frac{1}{2(1-\nu)} \frac{\partial}{\partial y}\left(\frac{\partial u_{c}}{\partial x}+\frac{\partial v_{c}}{\partial y}\right) \\
& +r_{1} \rho_{0}(x, y)\left(v_{0}-v_{c}\right)=0
\end{aligned}
$$

where $r_{1}$ represents ratio of effective substrate stiffness with respect to effective cell stiffness (see SI Sec. S2 for derivation), and $\nu$ ( $=0.45$ for near incompressibility) is the Poisson's ratio of the cell. The density of adhesion over cell area is denoted by $\rho_{0}(x, y)$. Figure $2 \mathrm{~b}$ shows a qualitative axially symmetric distribution of adhesion bonds over cell area. The value of cell displacements, $u_{c}$ and $v_{c}$ at equilibrium state (time $t=0$ ) were obtained by solving Eqs. 1 and 2 numerically by finite element analysis (FEA) using PDE toolbox of Matlab with stress free boundary conditions at the edge. Note that we have nondimensionalized all length-units with respect to $R_{\text {cell }}$ for compactness of equations (see SI for additional details).

The second stage or the de-adhesion stage was modeled by assuming a force independent first order kinetics with rate $r$ for bond breakage, beginning from the equilibrium configuration generated above, such that $\rho_{0}(x, y, t)=$ $\rho_{0}(x, y) \exp (-r t)$ was the bond density function at any 


$$
\begin{array}{r}
\frac{1}{2(1+\nu)}\left(\nabla^{2} u+\frac{\partial}{\partial t} \nabla^{2} u\right)+\frac{1}{2(1-\nu)} \\
\frac{\partial}{\partial x}\left(\frac{\partial u}{\partial x}+\frac{\partial v}{\partial y}\right)+\frac{1}{2(1-\nu)} \frac{\partial}{\partial t} \frac{\partial}{\partial x}\left(\frac{\partial u}{\partial x}+\frac{\partial v}{\partial y}\right) \\
+\rho_{0}(x, y) \exp \left(-r_{2} t\right)\left(r_{1}\left(u_{0}-u\right)-r_{3} \frac{\partial u}{\partial t}\right)=0 \\
\frac{1}{2(1+\nu)}\left(\nabla^{2} v+\frac{\partial}{\partial t} \nabla^{2} v\right)+\frac{1}{2(1-\nu)} \frac{\partial}{\partial y}\left(\frac{\partial u}{\partial x}+\frac{\partial v}{\partial y}\right)+\frac{1}{2(1-\nu)} \frac{\partial}{\partial t} \frac{\partial}{\partial y}\left(\frac{\partial u}{\partial x}+\frac{\partial v}{\partial y}\right) \\
+\rho_{0}(x, y) \exp \left(-r_{2} t\right)\left(r_{1}\left(v_{0}-v\right)-r_{3} \frac{\partial v}{\partial t}\right)=0
\end{array}
$$

The non-dimensionalization of all time-units was done with respect to $\tau_{0}$, which is the ratio of cell viscosity with cell stiffness. We also introduced two additional parameters $r_{2}$ - non-dimensionalized bond cutting rate and $r_{3}$ - the ratio of effective substrate viscosity to cell viscosity in these equations (see SI Sec. S2). The length scale for non-dimensionalization was $R_{\text {cell }}$ as before.

Taking the values of cell displacement at equilibrium state $\left(u_{c}\right.$ and $\left.v_{c}\right)$ from the solution of Eqs. 1 and 2 as the initial conditions, time dependent PDEs Eqs. 3 and 4 were solved to get the cell displacement values, $u(x, y, t)$ and $v(x, y, t)$, at any time $t$. To solve Eqs. 3 and 4 numerically, we again used Matlab PDE toolbox as earlier with ode15s solver for time integration. A sample discretized mesh used for the FEA is shown in Fig. 2b.

Our model could recover the sigmoidal behaviour for the dynamics of projected cell area as shown in Fig. 1b (see SI Fig. S2). We then focused on the theoretical understanding of translation and rotation motion of cell during de-adhesion process. To estimate the translation of cell, we tracked the displacement of the cell centroid as a function of time. The overall rotation of the cell at any time, $t$ was calculated as $\frac{\int \omega d A}{\int d A}$, where $\omega=\frac{1}{2}\left(\frac{\partial u}{\partial y}-\frac{\partial v}{\partial x}\right)$ is the rotation tensor, and $u$ and $v$ the cell displacements at the time $t$.

We investigated the role of contractility $\left(u_{0}(x, y), v_{0}(x, y)\right)$ and bond distribution $\left(\rho_{0}(x, y)\right)$ on cell translation and rotation during de-adhesion. In order to understand the influence of adhesion distributions on cell dynamics, three different types of bond distributions were considered in the study. To begin with, a fully symmetric adhesion distribution was applied throughout the cell contact area. In the second case, we took a bond distribution function with only one axis of symmetry (axially asymmetric bond distribution). Finally, completely asymmetric bond distribution (no axis of symmetry) was applied to the cell. In all the three cases, bond distribution was chosen in such a manner to generate highest number of adhesions at cell periphery and least at the centre, as is observed experimentally $\stackrel{10}{\underline{1}}$. Care had also been taken to keep the
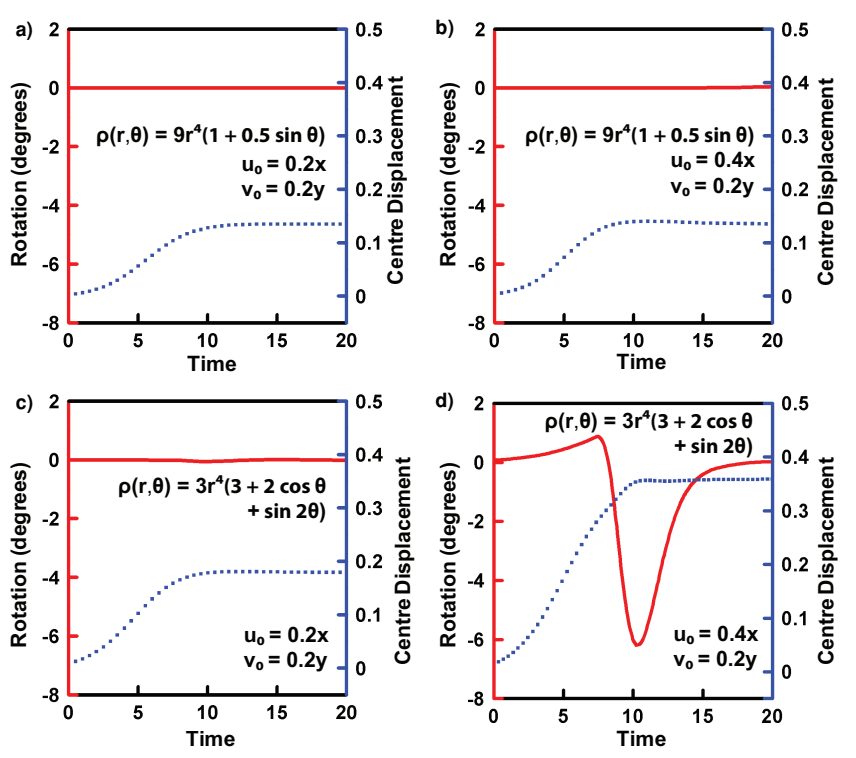

FIG. 3. (Color online) Effect of bond distribution and prestress on cell translation and rotation during de-adhesion. Plots between cell center displacement and rotation with time for various combination of bond distribution and prestress are shown. (a) Effect of axially asymmetric bond distribution on cell translation and rotation. (b) Combined effect of axially asymmetric bond distribution and anisotropic prestress on cell translation and rotation. (c) Effect of completely asymmetric bond distribution on cell translation and rotation. (d) Combined effect completely asymmetric bond distribution and anisotropic prestress on cell translation and rotation. For all these simulations values of non-dimensionalized parameters $r_{1}, r_{2}$ and $r_{3}$ were taken as $r_{1}=50, r_{2}=0.6$ and $r_{3}=0.1$; $\theta$ is as shown in Fig. 2 b.

total number of bonds $\int_{A} \rho_{0}(x, y) d A$ constant in all the cases. Similarly, for studying the effect of the nature of prestress on cell motions, we imposed two different types of prestress on cell; isotropic and anisotropic. Isotropic and anisotropic prestress were created by providing displacement fields, $u_{0}(x, y)$ and $v_{0}(x, y)$, of equal and 


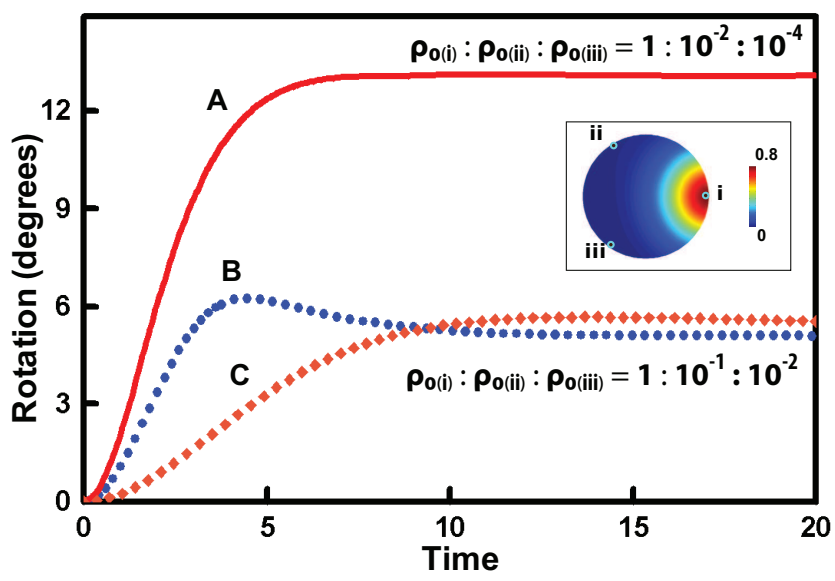

FIG. 4. (Color online) Role of bond distribution on cell rotation. (A), (B) and (C) show cell rotation with time for different values of bond density at three points $(i, i i$ and $i i i)$ on cell periphery, where the cell-substrate bonds were prominently concentrated. (A) Bond densities at $i$, ii and iii were significantly different (of the order of 100) from each other. (B), (C) The bond distribution function was same for both of these and the bond densities at $i, i i$ and $i i i$ were moderately different (of the order of 10) from each other. During the process of de-adhesion, bonds at all the three points were broken in a uniform manner for (A) and (B). For (C), only bonds concentrated at two points (ii and $i i i)$ were broken and the point with highest bond density $(i)$ remained intact (expressions for bond distribution function for (A), (B) and (C) are shown in SI Sec. S3). Inset showing the qualitative representation of bond densities at points $i$, ii and $i i i$ for (A), (B) and (C). For all these simulations, values of non-dimensionalized parameters were taken as $r_{1}=10, r_{2}=0.4$ and $r_{3}=0.1$, with $u_{0}=0.6 x$ and $v_{0}=0.2 y$.

unequal intensities along $x$ and $y$ direction, respectively, in the equilibration stage. Various combinations of bond distribution and prestress were applied to the cell and its translation and rotation upon de-adhesion were examined in each case. Throughout the simulations, the values of non dimensional parameters, $r_{1}, r_{2}$ and $r_{3}$ were kept constant.

After plotting cell centroid displacement and rotation with time, the following observations were made. If the cell-substrate bonds were symmetrically distributed, cell neither translated nor rotated during the de-adhesion process, irrespective of the nature of prestress. For an axially asymmetric distribution of bonds, translational motion occurred for both uniform and anisotropic prestress. Here we could not see cell rotation (Figs. [3a and 3b). Even when the bond distribution was completely asymmetric, cell did not rotate upon de-adhesion if the applied prestress was uniform in both directions (Fig. 3r). Finally, when the cell-substrate bonds were completely asymmetric and the applied prestress was non uniform, along with pure translation, cell exhibited some steering motion also (Fig. 3 d).

Although under the influence of anisotropic prestress and complete asymmetry in the bond distribution, the modeled cell exhibited a combination of translation and rotation, its behaviour did not mirror the experimental observation, where the observed rotation was largely monotonic (Fig. 1 d and Supp. Movie). Hence, as the next case, we tried a different type of bond heterogeneity and saw its effect on cell rotation. For that purpose, cell substrate bonds were assumed to be predominantly concentrated at three points on the cell periphery as opposed to the continuous of distribution of bonds in the earlier cases (SI Sec. S3) - prestress was also kept to be anisotropic. Figure 4 shows the plot of cell rotation with time for three different bond densities $\rho_{0}$ at points $i$, ii and iii. From the plot, it can be observed that cell with non uniform values of bond densities at different points on its periphery exhibited monotonic rotation during the process of de-adhesion. It was also noted that, as the difference in bond densities between various points increased, the amount of cell rotation also increased (Fig. 44 versus Fig. 4B B).

In conclusion, our results indicate that cellular translation arises due to partial asymmetry about any axis in the bond distribution and(or) bond strength, with contractility dictating the magnitude of the final movement (Fig. 3). In contrast, cellular rotation requires a combination of radial asymmetries in both bond distribution as well as contractility, with sustained monotonic rotational movement requiring a highly polarized distribution of finite adhesion spots (Figs. 3 and 4). Since cell migration involves the co-ordinated formation and breakage of adhesions, therefore, the spatial heterogeneity in adhesion distribution and cytoskeletal organization can impact both random as well as directed migration depending on the extent of spatio-temporal coupling between these two types of structures. Despite the complex mechanochemistry regulating cellular movements, our simulated de-adhesion experiments and theory directly implicate anisotropy and heterogeneity of adhesion distribution and contractility as two of the important factors influencing the directional changes associated with cell motility.

Acknowledgement: MMI gratefully acknowledges financial support from Department of Science and Technology, India.

${ }^{1}$ A. J. Ridley, M. A. Schwartz, K. Burridge, R. A. Firtel, M. H. Ginsberg, G. Borisy, J. T. Parsons, and A. R. Horwitz, Science 302, 1704 (2003).

${ }^{2}$ R. J. Petrie, A. D. Doyle, and K. M. Yamada, Nat. Rev. Mol. Cell Biol. 10, 538 (2009).

${ }^{3}$ S. Sen and S. Kumar, Cell. and Mol. Bioeng 2, 218 (2009).

${ }^{4}$ A. D. Mesquita and H. B. Coda, Eng. Anal. Bond. Elem. 27, 885 (2003).

${ }^{5}$ H. Karcher, J. Lammerding, H. Huang, R. T. Lee, R. D. Kamm, and M. R. Kaazempur-Mofrad, Biophys. J. 85, 3336 (2003).

${ }^{6}$ U. S. Schwarz, T. Erdmann, and I. B. Bischofs, Biosystems 83, 225 (2006).

${ }^{7}$ C. M. Edwards and U. S. Schwarz, Phys. Rev. Lett. 107, 128101 (2011).

${ }^{8}$ S. Banerjee and M. Marchetti, Phys. Rev. Lett. 109, 108101 (2012). 
${ }^{9}$ S. Banerjee and M. Cristina Marchetti, New J. Physic. 15, 035015 (2013).
${ }^{10}$ B. Geiger, J. P. Spatz, and A. D. Bershadsky, Nat. Rev. Mol. Cell Biol. 10, 21 (2009). 